\title{
CONDITIONS FOR THE COMMUTATIVITY OF SEMIGROUPS
}

\author{
G. KOWOL
}

\begin{abstract}
ABSTRACr. Let $S$ be a semigroup. Then by a theorem of Tully [7]: $S$ is a commutative semigroup iff $a b=b^{n} a^{m}$ for all $a, b \in S$ (m,n $\geqslant 1$, fixed). We prove the following: $S$ is a commutative semigroup iff $a b=b^{n(a, b)} a^{m(a, b)}$ for all $a, b \in S$, where one of the exponents $n(a, b)$ and $m(a, b)$ is constant and the other is independent of $a$ or $b$.
\end{abstract}

Tamura formulated in [6] the following question: Let $S$ be a semigroup and $f(x, y)$ a fixed word in $x, y \in S$; when does $a b=f(a, b)$ for all $a, b \in S$ imply the commutativity of $S$ ? He proved the following result:

Let $f(a, b)$ be $a$ word involving both $a$ and $b$, and let the length of $f(a, b)$ be greater than 2. $a b=f(a, b)$ implies $a b=b a$ in semigroups iff

(i) $f(a, b)$ starts in $b$ and ends in $a$, and

(ii) $a b=f(a, b)$ implies $a b=b a$ in groups.

Thus he obtained in particular: $a b=b^{n} a^{m}$ for all $a, b \in S$ and fixed $m, n \in N$ ( $N$ denotes the set of all natural numbers) implies the commutativity of $S$ (Tully [7]).

Putcha and Weissglass [5] generalized Tamura's problem, supposing that $f(x, y)$ is not necessarily a fixed word but can vary with $x$ and $y$. We prove the following

THEOREM. Let $S$ be a semigroup. Then the following conditions are equivalent:

(i) $S$ is commutative.

(ii) $a b=b^{n} a^{m(a)}$

(iii) $a b=b^{n} a^{m(b)}$

(iv) $a b=b^{n(a)} a^{m}$

(v) $a b=b^{n(b)} a^{m}$

for all $a, b \in S$ and exponents in $N$ ( $m, n$ are fixed and the other exponents depend only on the indicated variable).

REMARK. Note that conditions (iii) and (iv) and also (ii) and (v) are "dual": Assume for instance that condition (iii) holds in $S$. Then defining in $S$ the dual operation “。” (see e.g. Ljapin [2, p. 21]): $a \circ b=b a$, (iii) turns to $b \circ a$ $=a^{m(b)} \circ b^{n}$, thus (iv) holds in $(S, \circ)$. Therefore we need only prove that (ii) resp. (iii) implies (i)-the other cases are trivial.

First we show the proposition (ii) resp. (iii) $\Rightarrow$ (i) for the special case of groups, which generalizes Satz 2 of Lausch, Nöbauer and Schweiger [3]:

Received by the editors June 4, 1975.

AMS (MOS) subject classifications (1970). Primary 20M10.

○ American Mathematical Society 1976 
Lemma. Let $G$ be a group. Then the conditions (i)-(v) of the Theorem are equivalent.

Proof. (ii) $\Rightarrow$ (i). Fixing $a=1$ we obtain $b=b^{n}$, fixing $b=1$ we get $a=a^{m(a)}$, together $a b=b a$.

(iii) $\Rightarrow$ (i). Setting $a=1$ in (iii) we get $b=b^{n}$, thus

$$
a b=b a^{m(b)}, \text { hence } b^{-1} a b=a^{m(b)} \quad \text { for all } a, b \in G .
$$

Now let $U$ be an arbitrary subgroup of $G$; then (1) implies $b^{-1} U B$ $=U$ for all $b \in G$. This means that every subgroup $U$ of $G$ is normal in $G$.

Suppose now that $G$ is not abelian; then $G$ is hamiltonian and contains the quaternion group $Q$ as subgroup (see e.g. Hall [1, Chapter 12.5]). Evidently $Q$ satisfies condition (iii), since $G$ does. $Q$ is a nonabelian group of order 8 defined by $g^{4}=1, g^{2}=h^{2}, h^{-1} g h=g^{-1}$-hence the order of the center of $Q$ is 2 . By assumption $g^{-1} x g=x^{m(g)}$ for all $x \in Q$. Taking $x=g$ we get $g=g^{m(g)}$, thus $m(g) \equiv 1(\bmod 4(=o(g)=\exp Q))$. Therefore $g^{-1} x g$ $=x$ for all $x \in Q$, which means $g \in Z(Q)$, which is a contradiction to $o(Z(Q))=2$. Thus $G$ is abelian.

ProOF OF THE TheOREM. To show (ii) resp. (iii) $\Rightarrow$ (i) we can assume $n=1$ in both cases, since otherwise we apply the theorem of Putcha and Weissglass [5] (see also the remark on p. 67) and obtain that $S$ is an inflation of a semilattice of periodic groups $G_{i}$ (for the definitions see, e.g., Petrich [4]). Since $G_{i} \subseteq S$ and the conditions (ii) and (iii) are hereditary to subsemigroups, we get by the Lemma that $G_{i}$ is abelian for every $i$. Now an inflation of a semilattice of abelian groups is abelian again (see also Tamura [6]).

(ii) $\Rightarrow$ (i). Let $S$ satisfy $a b=b a^{m(a)}$ for all $a, b \in S$. We state for arbitrary $k$

$$
a^{k} b=b a^{k m(a)} \quad \text { for all } a, b \in S .
$$

The case $k=1$ is verified by condition (ii), and now proceeding by induction we obtain

$$
a^{k+1} b=a\left(b a^{k m(a)}\right)=(a b) a^{k m(a)} \stackrel{(\mathrm{ii})}{=} b a^{m(a)} a^{k m(a)}=b a^{(k+1) m(a)} .
$$

Now take an element $c$ with $n(c)>1$ and $d$ arbitrary in $S$. Putting first $d=a, c=b$ and then $c=a, d^{m(d)}=b$ in condition (ii) we get

$$
d c=c d^{m(d)}=d^{m(d)} c^{m(c)} .
$$

Applying (2) with $d=a, m(d)=k, c^{m(c)}=b$ we obtain finally

$$
d c=d^{m(d)} c^{m(c)}=c^{m(c)} d^{(m(d))^{2}} .
$$

On the other hand, we get by condition (ii), taking first $c=a, d$ $=b$ and then $d=a, c^{m(c)}=b$

$$
c d=d c^{m(c)}=c^{m(c)} d^{m(d)} .
$$

To conclude the proof we have only to show that $d^{m(d)}=d^{(m(d))^{2}}$ for all $d \in S$. Now fixing $a=b=d$ in condition (ii) we obtain $d^{2}=d^{m(d)+1}$; 
if $m(d)=2$ it follows $d^{2}=d^{3}=d^{4}=\cdots=d^{m(d)}=\cdots=d^{(m(d))^{2}}$. Now let $m(d)>2$, then $d^{2}=d^{m(d)+1}$ implies

$$
d^{m(d)}=d^{2} d^{m(d)-2}=d^{m(d)+1} d^{m(d)-2}=d^{2 m(d)-1}
$$

and also that $d^{2}, \ldots, d^{m(d)}$ form a group with the unity $d^{m(d)-1}$, as is well known. Therefore we obtain

$$
d^{m(d)}=d^{2 m(d)-1}=d^{2 m(d)-1}\left(d^{m(d)-1}\right)^{k} \quad \text { for arbitrary } k .
$$

Taking $k=m(d)-1$ we get the desired result: $d^{m(d)}=d^{(m(d))^{2}}$. Thus $S$ is commutative.

(iii) $\Rightarrow$ (i). Let $S$ satisfy $a b=b a^{m(b)}$ for all $a, b \in S$. We define

$$
I=\left\{b \in S, m(b)>1 \text { with } a b=b a^{m(b)} \text {, for all } a \in S\right\} .
$$

We show that $I$ is a subsemigroup of $S$ (it is easy to verify that $I$ is even a prime ideal of $S$ ). Let $s, t \in I$ arbitrary, then

$$
a s=s a^{m(s)} \text { and } a t=t a^{m(t)}
$$

for all $a \in S$. We first state for arbitrary $k$ and $a \in S$

$$
(a s)^{k}=s^{k} a^{l} \quad \text { with } l=m^{k}(s)+m^{k-1}(s)+\cdots+m(s) .
$$

This is proved by induction on $k$; the case $k=1$ is verified by (3). Now

$$
(a s)^{k+1}=\left(s^{k} a^{l}\right)(a s)=s^{k}\left(a^{l+1} s\right) \stackrel{(3)}{=} s^{k}\left(s a^{(l+1) m(s)}\right)=s^{k+1} a^{\bar{l}}
$$

with $\bar{l}=(l+1) m(s)=m^{k+1}(s)+m^{k}(s)+\cdots+m(s)$ concludes the proof of (4).

Applying equations (3) and (4) we get, therefore,

$$
(a s) t=t(a s)^{m(t)}=t s^{m(t)} a^{m(s, t)}
$$

with certain $m(s, t)>1$. Now putting $a=s$ in the second equation of (3) we obtain $s t=t s^{m(t)}$; together with (5) follows ast $=s t a^{m(s, t)}$ with $m(s, t)$ $>1$ for all $a \in S$, hence $I$ is a subsemigroup of $S$. By definition of $I$, therefore, $a b=b a^{m(b)}$ with $m(b)>1$ specially for $a, b \in I$. Now we can apply the above mentioned theorem of Putcha and Weissglass [5] and obtain again that $I$ is commutative. If we take finally an element $c \in S-I$, then $c$ is in the center of $S$ evidently; thus again $S$ is commutative.

REMARK . The Theorem cannot be sharpened in the following sense: $S$ is a commutative semigroup iff $a b=b^{n(a, b)} a^{m(a, b)}$ for all $a, b \in S$ with one exponent (even) constant and the other dependent on $a$ and $b$. A counterexample is given by the quaternion group $Q$ : since all subgroups of $Q$ are normal, we get $g^{-1} a g=a^{m(a, g)}$ for all cyclic subgroups $\langle a\rangle$ specially. Consequently $a g=g a^{m(a, g)}$ is satisfied for all $a, g \in Q$, but $Q$ is not abelian.

\section{REFERENCES}

1. M. Hall, Jr., The theory of groups, Macmillan, New York, 1959. MR 21 \# 1996.

2. E. S. Ljapin, Semigroups, Fizmatgiz, Moscow, 1960; English transl., Transl. Math. Monographs, vol. 3, Amer. Math. Soc., Providence, R. I., 1963. MR 22 \# 11054; 29 \# 4817. 
3. H. Lausch, W. Nöbauer and F. Schweiger, Polynompermutation n auf Gruppen, II, Monatsh. Math. 70 (1966), 118-126. MR 33 \# 1356.

4. M. Petrich, Introduction to semigroups, Merrill, Columbus, Ohio, 1973.

5. M. S. Putcha and J. Weissglass, Semigroups satisfying variable identities, Semigroup Forum 3 (1971/72), no. 1, 64-67. MR 44 \#6881.

6. T. Tamura, Semigroups satisfying identity $x y=f(x, y)$, Pacific J. Math. 31 (1969), 513-521. MR 41 \# 5528.

7. E. J. Tully, Semigroups satisfying an identity of the form $x y=y^{m} x^{n}$ (unpublished manuscript).

Department of Mathematics, University of Vienna, Vienna, Austria 